\section{neuro- psychiatrie}

vereinigt mit

psychiatrie \& psychotherapie

\title{
Bericht aus dem Vorstand der ÖGKJP
}

\author{
Andreas Karwautz, Kathrin Sevecke, Anna-Katharina Purtscher-Penz, Roland GraßI
}

(c) Springer-Verlag GmbH Austria, ein Teil von Springer Nature 2021

\section{Liebe Mitglieder, liebe \\ Kolleginnen, liebe Kollegen,}

Das Präsidium der ÖGKJP hat sich in den letzten 3 Monaten neben der so wichtigen und gut aufgenommenen Pressearbeit erneut intensiv um weitere Schritte zur Behebung des Versorgungsmangels in unserem Fach bemüht.

Zum einen fanden Gespräche mit der GÖG und der ÖÄK statt, um erneut die mangelhafte Ausgangslage bezogen auf zu erwartende Fachärzte, Ausbildungsstellen und Ausbildungsschlüssel $\mathrm{zu}$ klären.

Zum anderen hat das Präsidium mit Hilfe der Klinikleitungen mit Stichtag 1. April 2021 erneut die Ausbildungssituation in den Kliniken abgefragt, um so einen Vergleich zu der Erhebung mit Stichtag 31. Dezember 2019 herstellen zu können. An der Anzahl der bewilligten Ausbildungsstellen mit $n=96$ hat sich in den knapp 1,5 Jahren nichts geändert. Aktuell sind in den befragten Einrichtungen 103 Facharzt-Stellen bewilligt, aber aufgrund des Mangels an Fachärzten nur 92 Stellen besetzt. Die Erhebung von Rainer Fliedl ergab 100 besetzte Facharztstellen. Es lässt sich

Univ.-Prof. Dr. A. Karwautz ( $₫)$

Universitätsklinik für Kinder- und

Jugendpsychiatrie, Medizinische

Universität Wien, Währinger Gürtel 18-20, 1090 Wien, Österreich

andreas.karwautz@meduniwien.ac.at also eindeutig festhalten, dass es in den letzten 1,5 Jahren nicht zu einer besseren fachärztlichen Ausstattung an den Kliniken in unserem Fach gekommen ist (Fliedl R, Ecker B \& Karwautz A. Kinder- und jugendpsychiatrische Versorgung 2019 in Österreich - Stufen der Versorgung, Ist-Stand und Ausblick. Neuropsychiatr 2020;34:179-188). Dies ist vor dem Hintergrund der deutlich gestiegenen Inanspruchnahme - u.a. durch die Pandemie - als dramatisch zu bewerten. Diesbezügliche Lösungsschritte auf mehreren Ebenen werden in den nächsten Monaten mit der ÖÄK und dem Ministerium diskutiert. Eine entsprechende Diskussionsvorlage wurde ausgearbeitet.

Erfreulicherweise wurde die in einer 1. Novelle der ÄAO 2015 für unser Fach wichtige Fristverlängerung von 2019 auf 2024 bezüglich $\S 30$ Fachärztinnen/ Fachärzten für Kinder- und Jugendpsychiatrie mit Diplom Psychotherapeutische Medizin vorgenommen. Diese für uns wichtige Änderung wurde durch BM Rudolf Anschober umgehend mittels einer Novelle umgesetzt, ebenso die Verlängerung der Mangelfachregelung bis 2027. Wir haben dem scheidenden $\mathrm{BM}$ ein Dankesschreiben für die wirklich ehrlichen Bemühungen um den dringend notwendigen Ausbau unseres Fachgebietes gesendet. Ebenso hat der neue BM, Dr. Wolfgang Mückstein, am ersten Arbeitstag ein Gratulationsschreiben erhalten, mit der Bitte um einen Gesprächstermin verbunden mit dem Wunsch den schon begonnenen Dialog konstruktiv fortzusetzen, da ja noch viele Problembereiche mit Unterstützung des Gesundheitsministeriums angegangen werden müssen.

Wir freuen uns sehr über zwei neue Primariate für KJP, zum einen in Schwarzach-St. Veit (Prim. Dr. Alexander Holzknecht) und zum anderen in Wels-Grieskirchen (Prim. Dr. Adrian Kamper).

Wir weisen auch nochmals darauf hin, dass mit 1. Februar 2021 Frau Dr. Belinda Plattner die Professur für KJP an der PMU Salzburg und die Klinikleitung an der SALK-Abteilung für KJP als Nachfolgerin von Herrn Prof. Dr. Leonhard Thun Hohenstein übernommen hat. Wir wünschen den 3 Abteilungsleitern alles Gute!

Referentin: Kathrin Sevecke

\section{Ethikbeirat der ÖGKJP}

Die Behandlung von Kindern und Jugendlichen mit psychischen Erkrankungen erfordert, über die grundsätzliche Schutzwürdigkeit von Kindern und Jugendlichen hinaus, eine besondere Sensibilität gegenüber den Patient*innen und ihren Familien. Es geht um die Wahrung der grundsätzlichen Rechte der Autonomie und Partizipation von Minderjährigen an Therapieentscheidungen, Respekt vor dem Wunsch der Patient*innen und die Transparenz bei Entscheidungen und Handlungsabläufen 
im Rahmen der Behandlung. Die bestmögliche Aufklärung der Kinder und Jugendlichen sowie deren Obsorgeberechtigten schaffen die notwendigen Voraussetzungen dafür.

Die besondere Beziehung zwischen Arzt/Ärztin und Patient/Patientin im Rahmen der ärztlichen Behandlung und Durchführung der psychotherapeutischen Medizin muss entsprechende Würdigung erfahren: Durch Achtung und Wahrung der Wünsche, Ansichten und Hoffnungen der Patient*innen und durch die Sorge um die Aufrechterhaltung ihrer physischen und psychischen Integrität im gesamten Verlauf der Behandlung.

Die Einhaltung dieser Grundsätze stellt eine ethische Anforderung an alle Mitglieder der Österreichischen Gesellschaft für Kinder- und Jugendpsychiatrie, Psychosomatik und Psychotherapie dar.

Zur Anhörung von Fragen von Patient*innen, Obsorgeberechtigten oder Kolleg*innen und zur Klärung ev. aufkommender Fragestellungen oder Beschwerden wurde von der ÖGKJP nunmehr ein Ethikbeirat eingerichtet. Der Beirat verpflichtet sich der Beratung der Anfragenden und informiert über Beratungsmöglichkeiten durch zuständige Institutionen. Darüber hinaus ist der Ethikbeirat auch ein beratendes Organ des Vorstandes der ÖGKJP. Bei Fragestellungen und Beschwerden soll der Ethikbeirat ein größtmögliches Maß an Objektivität gewährleisten. Die konstituierende Sitzung des Ethikbeirats fand unter der Leitung von Frau Prim. ${ }^{a}$ Purtscher-Penz am 24. März 2021 statt.

Referentin: Anna-Katharina PurtscherPenz

\section{Ausbildungskommission}

2021 wurden bereits zwei CuPsy-Module (Adoleszenz und Familienarbeit) erfolgreich als Webinare abgehalten. Weiters fanden die ersten beiden Wahlmodule des CuPsy (Psychodrama, IPT) mit organisatorischer Unterstützung der Ausbildungskommission statt. Zum jetzigen Zeitpunkt ist geplant, spätestens im Wintersemester wieder zu den Präsenzveranstaltungen in Salzburg wie gewohnt zurück zu kehren.

Weiters beginnt am 27. Mai 2021 und 24. Juni 2021 unsere Veranstaltungsserie „KJPP-Crossover“ mit zweistündigen Abendveranstaltungen zu Themen im Grenzbereich der KJPP bzw. mit Perspektiven aus benachbarten Disziplinen wie Psychologie, Soziologie, Recht, usw. Gegen einen Unkostenbeitrag von EUR 10,00 sollen 4 Abende pro Jahr angeboten werden. Auch der vielfach geäußerte Wunsch nach vermehrter Aus- und Fortbildung im forensischen Bereich soll hier erfüllt werden.

Die AssistentInnen-Tage werden heuer am 22. September 2021 (ÖGKJPKongress Bad Tatzmannsdorf, Psychopharmakologie - Prof. Gerlach, PD Taurines, Würzburg) und am 20. Oktober 2021 (Symposium Pöllau, Kommunikation und Teamprozesse) hoffentlich als Präsenzveranstaltung stattfinden.

Auch die Fachwerkstatt-Gösing soll in Präsenz von 8. bis 9. Otkober 2021 stattfinden und wird das Thema „Geschwister" behandeln.

Aus ausbildungspolitischer Sicht arbeitet der Vorstand geschlossen nach wie vor an den Bedingungen, mehr Kolleginnen und Kollegen ausbilden zu dürfen. Dies ist beharrliche Überzeugungsarbeit in ÖÄK-Gremien und im BMGSPK.

Referent: Roland GraßI

\section{Terminankündigungen}

Es ist uns eine große Freude, Sie wieder zum Jahreskongress der ÖGKJP vom 23. bis 25. September 2021 nach Bad Tatzmannsdorf und zum 21. Kinderund Jugendpsychiatrischen Symposium in Schloss Pöllau vom 21. bis 23. Oktober 2021 einladen zu können.

Wir sind froh, dass die Veranstaltungen auf jeden Fall stattfinden werden. Voraussetzung sind selbstverständlich die dann aktuellen COVID-19-Bestimmungen mit den entsprechenden Hygiene-Konzepten.

Wir bitten Sie schon jetzt um Verständnis für diese Regelungen und ersuchen Sie um Ihre Mithilfe bei der Umsetzung am Veranstaltungsort.

Weitere Informationen: https:// www.lkh-graz2.at/fileadmin/media/lkhgraz2/Bilder/KJP/KJP_2021_21_KJP_ Symposium_Poellau.pdf

Der 12. Wiener Kongress Essstörungen wird vom 17. bis 19. März 2022 in Wien stattfinden. Der 11. Kongress wurde als reine Onlineveranstaltung im März 2021 mit sehr großem Erfolg durchgeführt, das virtuelle Format wurde gut angenommen, es konnten dadurch auch über 100 Teilnehmer aus Deutschland, der Schweiz und Südtirol teilnehmen.

Mit kollegialen Grüßen,

für die Autoren und den Vorstand

Univ.-Prof. Dr. Andreas Karwautz

Hinweis des Verlags Der Verlag bleibt in Hinblick auf geografische Zuordnungen und Gebietsbezeichnungen in veröffentlichten Karten und Institutsadressen neutral. 